Documento No. 23

Distribución del Financiamiento Otorgado por el Sistema Bancario Mexicano a la Banca Privada y Mixta

Por

Víctor M. Guerrero y Gabriel Vera Ferrer

Julio, 1980

Las ideas contenidas en el presente ensayo son responsabilidad exclusiva de los autores y no reflejan la posición del Banco de México, S.A. 


\title{
DISTRIBUCIÓN DEL FINANCIAMIENTO OTORGADO POR EL SISTEMA BANCARIO \\ MEXICANO A LA BANCA PRIVADA Y MIXTA
}

por

\author{
Víctor M. Guerrero y Gabriel Vera Ferrer
}

\section{Introducción}

Este trabajo tiene dos objetivos fundamentales:

1. Estudiar y cuantificar la estacionalidad mensual de la serie del financiamiento otorgado por el sistema bancario mexicano a la banca privada y mixta, y

2. Proponer un mecanismo para efectuar la distribución del financiamiento a lo largo del año, el cual tome en consideración y mantenga la estacionalidad observada en la serie histórica.

El procedimiento que se utiliza para lograr estos objetivos es el descrito en el documento de Vera y Guerrero (1980). En el presente estudio se formalizan más los pasos sugeridos en aquel documento y se confirma empíricamente la validez de los mismos.

\section{II. $\quad$ Ajuste Estacional de la Serie}

Como una primera etapa para realizar el ajuste estacional de la serie bajo estudio, cuyos datos aparecen en la tabla 1, se le aplicó la prueba del mes adyacente. Dicha prueba consiste en obtener promedio mensuales de los cocientes de variación de un mes respecto a la media aritmética de los valores del mes precedente y el mes siguiente (esta prueba se describe en Makridakis y Weelwright, 1978). Si esos promedio mensuales se acercan a la unidad, digamos que están dentro del intervalo $(0.95,1.05)$, se concluye que la estacionalidad mensual no es significativa.

Dado que los valores de los promedios de cocientes de variación caen dentro del intervalo $(0.9927,1.0059)$, se obtiene la conclusión de que la estacionalidad no es relevante para la serie. 
Tabla 1

Financiamiento otorgado por el Sistema Bancario Mexicano a la Banca Privada y Mixta

(Saldos en millones de pesos)

\begin{tabular}{|c|c|c|c|c|c|c|c|c|c|c|c|c|}
\hline AÑO & ENERO & FEBRERO & MARZO & ABRIL & MAYO & JUNIO & JULIO & AGOSTO & SEPTIEMBRE & OCTUBRE & NOVIEMBRE & DICIEMBRE \\
\hline 1970 & $73,367.80$ & $74,228.00$ & $75,210.10$ & $76,841.50$ & $77,940.70$ & $79,709.30$ & $81,419.70$ & $82,816.70$ & $84,108.00$ & $86,084.40$ & $88,250.90$ & $90,083.00$ \\
\hline 1971 & $90,533.70$ & $91,732.80$ & $92,812.20$ & $92,430.70$ & $93,844.80$ & $95,423.40$ & $96,194.70$ & $97,140.50$ & $98,042.60$ & $98,651.00$ & $99,691.00$ & $100,207.80$ \\
\hline 1972 & $100,756.10$ & $100,959.20$ & $100,695.50$ & $101,740.40$ & $102,960.20$ & $104,792.80$ & $106,262.50$ & $108,132.10$ & $109,179.80$ & $110,982.60$ & $112,221.20$ & $115,351.60$ \\
\hline 1973 & $115,179.10$ & $116,429.30$ & $118,115.60$ & $118,862.70$ & $121,162.20$ & $122,910.00$ & $124,664.40$ & $126,556.80$ & $128,186.00$ & $127,131.20$ & $127,018.10$ & $126,262.50$ \\
\hline 1974 & $128,745.80$ & $126,637.80$ & $127,552.60$ & $128,448.00$ & $132,217.40$ & $133,541.40$ & $135,270.30$ & $136,428.10$ & $138,129.80$ & $138,117.50$ & $139,797.40$ & $140,992.80$ \\
\hline 1975 & $140,521.20$ & $140,865.50$ & $142,101.50$ & $143,672.90$ & $149,068.90$ & $151,316.70$ & $153,222.40$ & $155,510.70$ & $157,696.60$ & $161,652.80$ & $164,363.00$ & $166,823.80$ \\
\hline 1976 & $167,525.80$ & $168,671.10$ & $171,420.70$ & $175,031.70$ & $179,188.60$ & $179,457.00$ & $183,039.10$ & $183,286.10$ & $183,837.10$ & $186,317.90$ & $188,045.50$ & $187,985.10$ \\
\hline 1977 & $188,789.90$ & $188,708.30$ & $193,390.30$ & $196,346.40$ & $202,206.70$ & $204,920.20$ & $206,367.00$ & $207,302.20$ & $212,902.00$ & $217,458.30$ & $222,114.50$ & $232,428.30$ \\
\hline 1978 & $230,737.70$ & $232,871.20$ & $239,940.50$ & $24,458.50$ & $253,106.90$ & $264,628.70$ & $273,490.20$ & $281,864.40$ & $291,410.90$ & $298,380.90$ & $305,753.60$ & $309,968.70$ \\
\hline 1979 & $316,904.00$ & $323,088.30$ & $331,885.60$ & $335,593.50$ & $351,782.20$ & $361,415.40$ & $366,874.30$ & $378,330.30$ & $387,480.20$ & $401,280.00$ & $411,639.30$ & $422,619.30$ \\
\hline
\end{tabular}

Esta conclusión está respaldada por la figura 1, ya que visualmente no parece existir estacionalidad mensual; de igual manera, en la Tabla 2 se muestran los factores de estacionalidad obtenidos con el procedimiento del Census Method II (CM-II), los cuales, al ser muy cercanos a la unidad, justifican la duda de que la estacionalidad sea significativa. 


\section{Gráfica 1}

Gráfica de la Serie del Financiamiento otorgado a la Banca Privada y Mixta

Miles de Millones de Pesos

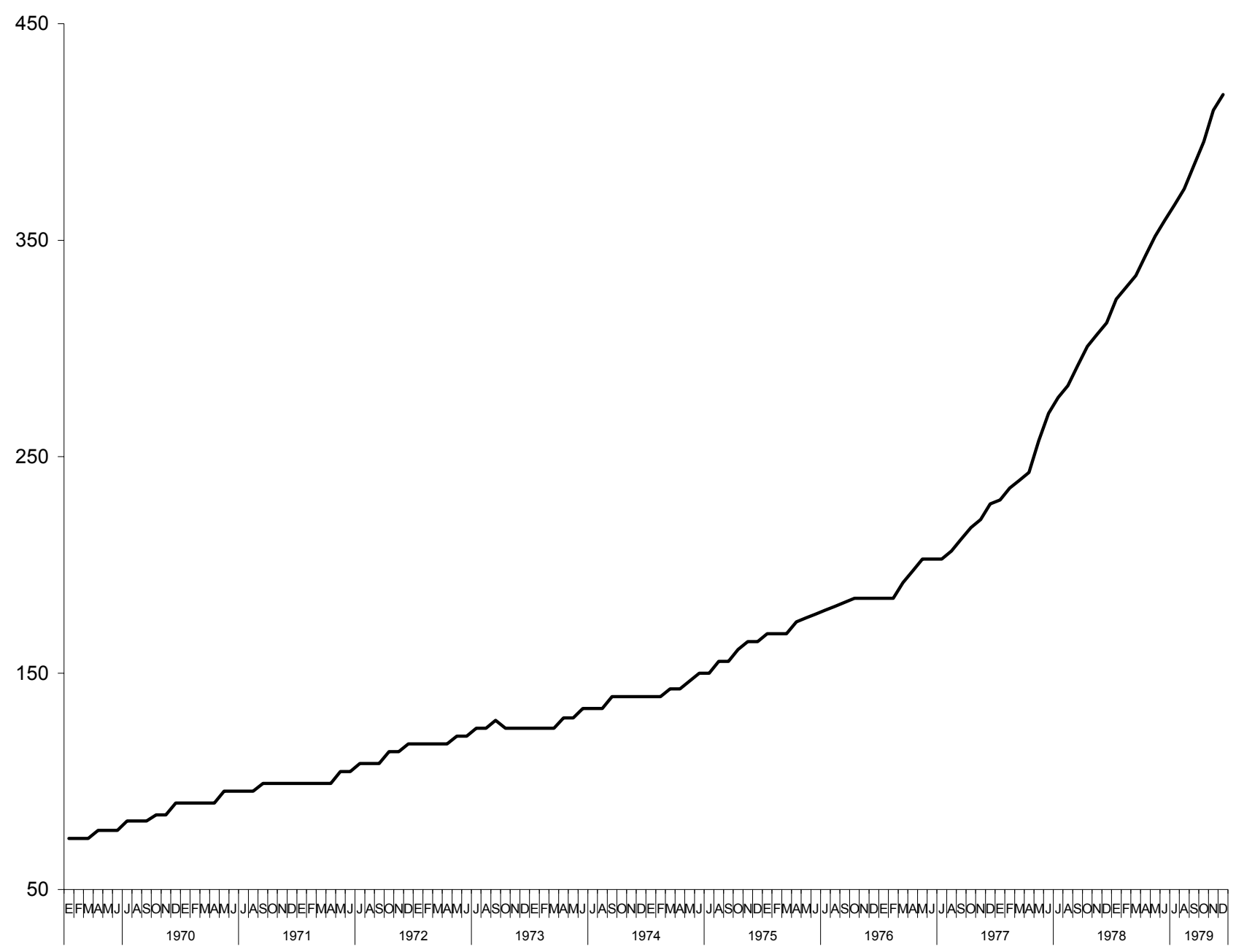


Tabla 2

Factores de Estacionalidad

\begin{tabular}{cccccccccccc}
\hline Ene. & Feb. & Mar. & Abr. & May. & Jun. & Jul. & Ago. & Sep. & Oct. & Nov. & Dic \\
\hline .9917 & .9865 & .9878 & .9870 & .9984 & 1.0036 & 1.0062 & 1.0057 & 1.0074 & 1.0076 & 1.0086 & 1.0096 \\
\hline
\end{tabular}

Puesto que las autocorrelaciones espurias pueden se la causa de que la estacionalidad no se vea claramente, se procede a eliminar dichas autocorrelaciones o, equivalentemente, se procede a buscar una función de los datos para la cual sí sea relevante la estacionalidad.

El procedimiento seguido consiste esencialmente en determinar los valores $\delta$ y d para los cuales se tenga que

$$
(1-B)^{d} Z_{12 t+q}^{1-\delta}=\mathrm{I}_{\mathrm{q}} X_{12 t+q}, \mathrm{t}=0,1, \ldots, \mathrm{m}-1 ; \mathrm{q}=1, \ldots, 12
$$

donde $\left\{Z_{12 \mathrm{t}+\mathrm{q}}\right\}$ es la serie original, $\left\{\mathrm{X}_{12 \mathrm{t}+\mathrm{q}}\right\}$ es una nueva serie que se puede representar por un modelo ARMA (véase Box y Jenkins, 1970), $\mathrm{I}_{\mathrm{q}}$ es el índice de estacionalidad correspondiente al mes $\mathrm{q}$, $\left(\sum_{q=1}^{12} \mathrm{I}_{\mathrm{q}}=12\right)$ y B es un operador de retraso, tal que

$$
B^{d} Z_{12 t+q}^{1-\delta} Z_{12 t+\mathrm{q}-\mathrm{d}}^{1-\delta}, \quad \mathrm{d}=0,1, \ldots
$$

Esto significa que la serie para la cual es relevante la estacionalidad es

$$
\left\{(1-B)^{d} Z_{12 t+q}^{-1-\delta}\right\}
$$

$\mathrm{y}$, por consiguiente, $\left\{\mathrm{X}_{12 \mathrm{t}+\mathrm{q}}\right\}$ es la versión desestacionalizada de dicha serie.

Primeramente se determina el valor de $\delta$ para el cual la serie $\left\{Z_{12 t+q}^{1-\delta}\right\} \underline{\text { muestre varianza }}$ aproximadamente constante; esto se logra con una aplicación del método de Bartlett (1947), para lo cual se elaboró la Tabla 3, en donde se observa que el mínimo coeficiente de variación se obtiene para $\delta=3 / 2$. Lo anterior indica que la potencia por utilizar debe ser $1-\delta=-1 / 2$, o sea que la transformación adecuada para estabilizar la varianza es el recíproco de la raíz cuadrada. 
Una vez fijado el valor de $\delta$, se procede a determinar el valor del parámetro d de tal manera que

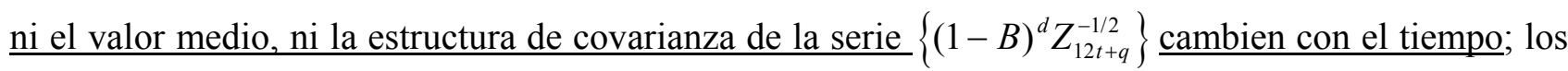
valores de $\mathrm{d}$ que se ensayan son $\mathrm{d}=0,1,2$ y 3 (nótese que $\mathrm{d}=0$ implica que el operador $(1-\mathrm{B})^{\mathrm{d}}$ no cambia la serie $\left\{Z_{12 t+q}^{-1 / 2}\right\}$ ). Con el fin de poder visualizar más claramente el efecto de d, se amplificó la escala multiplicando todos los valores de la serie por el facto $10^{5}$. Se puede observar, en la Figura 2, que la primera diferencia $(\mathrm{d}=1)$ satisface de manera aproximada el criterio para seleccionar $\mathrm{d}$. Por tanto, se tiene que es en la serie $\left\{10^{5}(1-B) Z_{12 t+q}^{-1 / 2}\right\}$ en donde la estacionalidad puede ser significativa.

Tabla 3

Coeficientes de Variación para diferentes Valores de $\delta$

\begin{tabular}{|c|c|c|c|c|c|}
\hline $\bar{\delta}$ & 0 & $1 / 2$ & 1 & $3 / 2$ & 2 \\
\hline AÑO & $\mathrm{S}_{\mathrm{Z}}$ & $\mathrm{S}_{\mathrm{Z}} / \mathrm{Z}^{1 / 2}$ & $\mathrm{~S}_{\mathrm{Z}} / \mathrm{Z}$ & $\mathrm{S}_{\mathrm{Z}} / \mathrm{Z}^{3 / 2}$ & $\overline{\mathrm{S}_{\mathrm{Z}} / \mathrm{Z}}$ \\
\hline 1970 & 5557.3237 & 19.54605 & 0.068747 & $2.4179407-04$ & $8.5043076-07$ \\
\hline 1971 & 3268.3079 & 10.57274 & 0.034202 & $1.1064127-04$ & $3.5791664-07$ \\
\hline 1972 & 5002.2804 & 15.35211 & 0.047116 & $1.4460003-04$ & $4.4378078-07$ \\
\hline 1973 & 4610.6438 & 13.16218 & 0.037575 & $1.0726555-04$ & $3.0621502-07$ \\
\hline 1974 & 5354.9238 & 14.65054 & 0.040082 & $1.0966161-04$ & $3.0002335-07$ \\
\hline 1975 & 9250.2074 & 23.70734 & 0.060759 & $1.5572038-04$ & $3.9909547-07$ \\
\hline 1976 & 7281.1063 & 17.18639 & 0.040567 & $9.5754558-05$ & $2.2601997-07$ \\
\hline 1977 & 13499.6354 & 29.73731 & 0.065506 & $1.4429826-04$ & 3.1786360 .07 \\
\hline 1978 & 28707.0560 & 55.36074 & 0.106762 & $2.0588668-04$ & $3.9704657-07$ \\
\hline 1979 & 35430.7224 & 58.59258 & 0.096896 & $1.6023889-04$ & 2.6499066 .07 \\
\hline $\begin{array}{l}\text { Coeficiente } \\
\text { de variación }\end{array}$ & 0.947902 & 0.672605 & 0.423039 & 0.316486 & 0.455534 \\
\hline
\end{tabular}

Para saber si existe realmente estacionalidad mensual en esta nueva serie, se le aplica la prueba del mes adyacente, la cual produce los promedios de cocientes de variación de la Tabla 4. De dichos promedios se obtiene la evidencia necesaria para suponer que la estacionalidad mensual es relevante. 
Gráfica 2

Función de Autocorrelación de la Primera Diferencia del Recíproco de la Raíz Cuadrada de la Serie

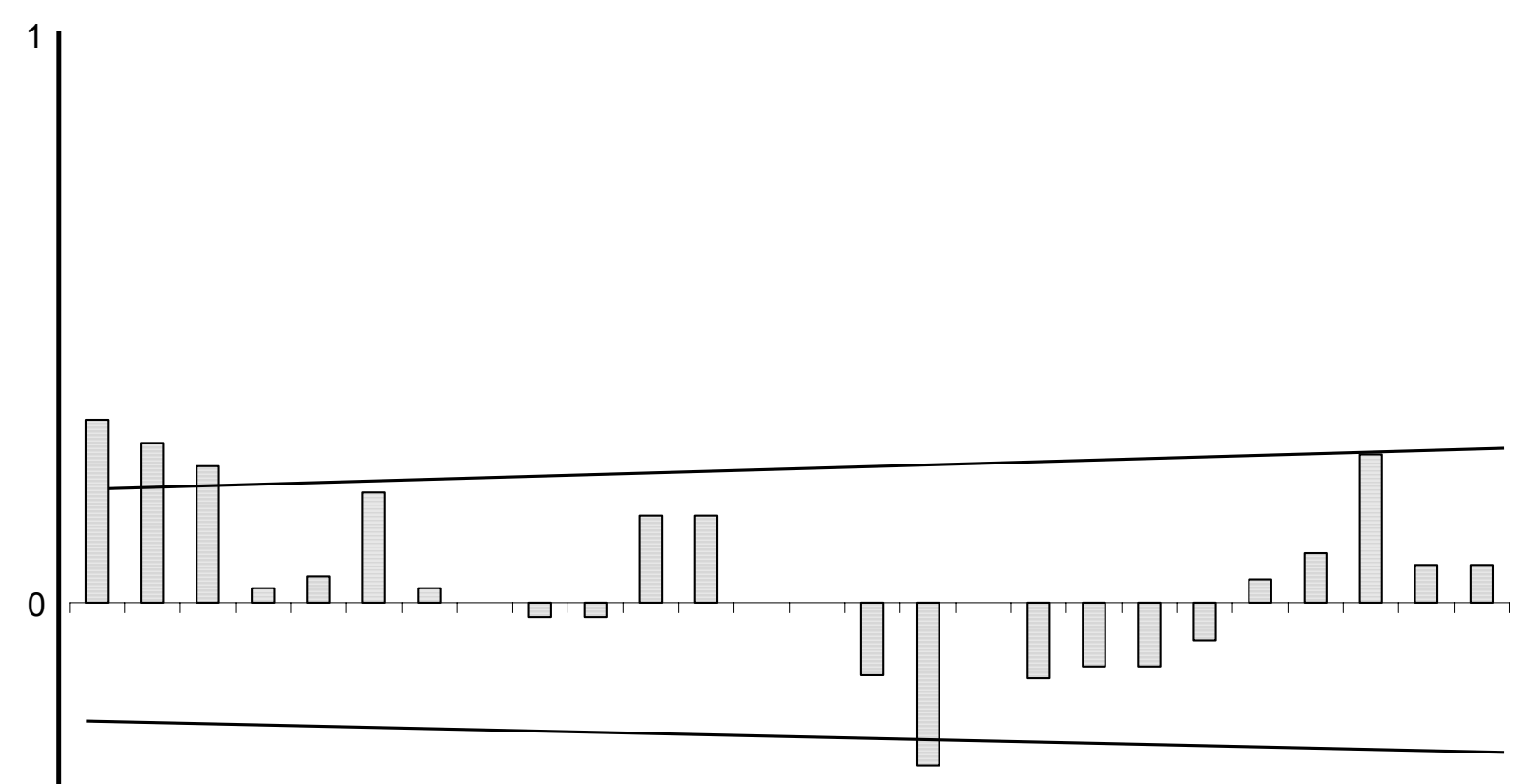

Tabla 4

Promedio de Cocientes de Variación para la Serie

$$
\left\{10^{5}(1-B) Z_{12 t+a}^{-1 / 2}\right\}
$$

\begin{tabular}{cccccccccccc}
\hline Ene. & Feb. & Mar. & Abr. & May. & Jun. & Jul. & Ago. & Sep. & Oct. & Nov. & Dic \\
\hline-0.7188 & 1.1316 & 1.5262 & 0.7268 & 1.9873 & 0.8965 & 2.1751 & 0.9330 & 1.4290 & 0.8908 & 1.507 & 2.5181 \\
\hline
\end{tabular}

Dada esta prueba, es razonable estimar, mediante el $\mathrm{CM}=\mathrm{II}$, los índices mensuales de estacionalidad Iq, $\left(q=1, \ldots, 12, \sum_{q=1}^{12} I_{q}=12\right)$, que se muestran en la Tabla 5. 
Debe notarse que estos índices se calculan bajo el supuesto de que la estacionalidad mensual es estable en el tiempo. Conforme a este supuesto, se obtiene la versión desestacionalizada de la serie como

$$
\left\{X_{12 t+q}\right\}=\left\{10^{5}(1-B) Z_{12 t+q}^{-1 / 2} / I q\right\}
$$

la cual se muestra gráficamente en la Figura 3, junto con la serie $\left\{10^{5}(1-B) Z_{12 t+q}^{-1 / 2}\right\}$.

\section{Tabla 5}

Indices de Estacionalidad de $\left\{10^{5}(1-B) Z_{12 t+q}^{-1 / 2}\right\}$

\begin{tabular}{|c|c|c|c|c|c|c|c|c|c|c|c|c|}
\hline & 1 & 2 & 3 & 4 & 5 & 6 & 7 & 8 & 9 & 10 & 11 & 12 \\
\hline$\overline{I_{q}}$ & 0.1763 & 0.5174 & 1.0193 & 0.8742 & 1.9441 & 1.3057 & 1.2526 & 1.0274 & 1.1410 & 0.8503 & 1.0127 & 0.8788 \\
\hline
\end{tabular}

Ahora bien, de acuerdo con (2.1), se debe encontrar un modelo de tipo ARMA que se adecue al comportamiento de la serie $\left\{X_{12 t+q}\right\}$. Esto se hace con la finalidad de obtener pronósticos de la serie desestacionalizada de manera eficiente. Sin embargo, no es necesario identificar un modelo para el caso presente, debido a que la serie desestacionalizada, $\left\{X_{12 t+q}\right\}$ puede considerarse como "ruido blanco", ya que el estadístico calculado Q de Box y Pierce (véase Box y Jenkins, 1970) toma el valor 27.969 , el cual debe compararse con valores de tablas de la distribución ji-cuadrada con 24 grados de libertad. Por tanto, la media aritmética de las observaciones desestacionalizadas $\bar{X}$, proporciona la representación que se buscaba del fenómeno, la cual permite obtener pronósticos para la serie $\left\{X_{12 t+q}\right\}$. 
Gráfica 3

Gráfica de la Serie $\left\{10^{5}(1-B) \mathrm{Z}_{121 \bullet q}^{-\frac{1}{2}}\right\}$ y de la Serie Desestacionalizada

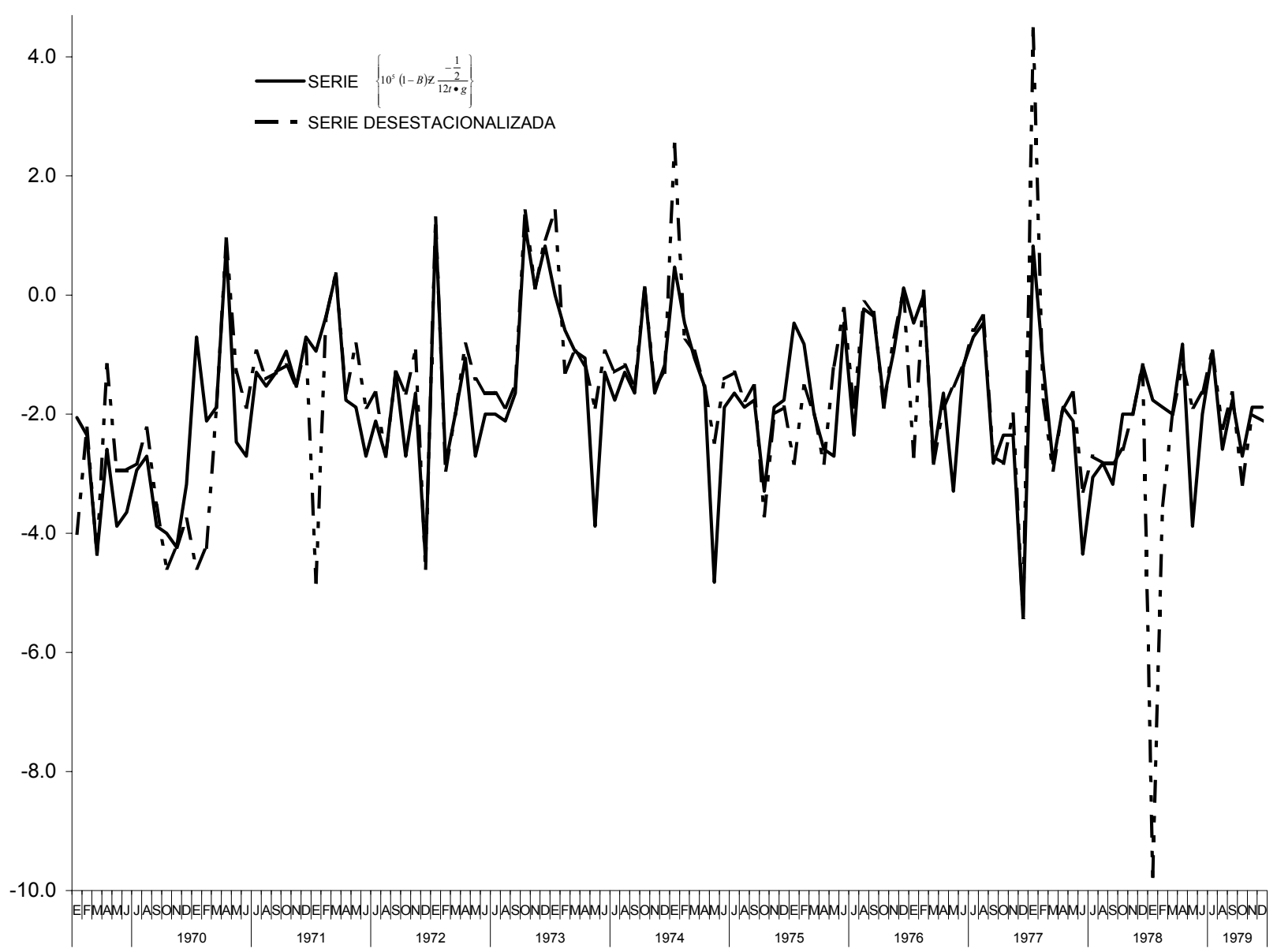

\section{Distribución del financiamiento}

La distribución del financiamiento se hará mediante el procedimiento sugerido por Vera y Guerrero. Dicho procedimiento se describirá aquí en términos del modelo (2.1) con el que se trabajó en la sección anterior, pero con la suposición adicional de que la serie $\left\{X_{12 t+q}\right\}$ que aparece en (2.1) es "ruido blanco" -la razón de este supuesto es que, tanto en la aplicación que aparece en Vera y Guerrero (1980; como en la presente, ese ha sido el caso. Así pues, en general el modelo estimado viene a ser

$$
(1-B)^{d} \hat{Z}_{12 t+q}^{1-\delta} \overline{\mathrm{X}} \cdot \mathrm{I}_{\mathrm{q}} \quad \text { si } \delta \neq 1
$$

$$
\mathrm{t}=0,1, \ldots \mathrm{m}-1 ; \mathrm{q}=1, \ldots 12
$$


$(1-B)^{d} \log \left(\hat{Z}_{12 t+q}\right)=\overline{\mathrm{X}} \cdot \mathrm{I}_{\mathrm{q}} \quad$ si $\delta=1$

el cual se utiliza para efectuar la distribución del financiamiento a lo largo del año.

Supóngase que el financiamiento ya ha sido otorgado hasta el mes $\mathrm{c}-1(1 \leq \mathrm{c}<12)$ del año $\mathrm{m}$, es decir, se conocen ya los valores de $\mathrm{Z}_{12 \mathrm{~m}+1},,, \mathrm{Z}_{12 \mathrm{~m}+\mathrm{c}-1}$, y se desea distribuir el financiamiento restante con el criterio de mantener la estacionalidad observada históricamente, para lo cual se requiere estimar $\mathrm{Z}_{12 \mathrm{~m}+\mathrm{c}}, \ldots \mathrm{Z}_{12 \mathrm{~m}+12}$. La restricción existente es entonces

$$
\mathrm{K}=\mathrm{Z}_{12 \mathrm{~m}+12}-\mathrm{Z}_{12 \mathrm{~m}+\mathrm{c}-1}
$$

con el valor de $K$, determinante del valor $Z_{12 m+12}$, fijado exógenamente.

Se consideran ahora tres casos que, según se piensa, tienen mayor posibilidad de ocurrir en la práctica; asimismo, los resultados se dan para $\delta \neq 1$, ya que para $\delta=1$, el único cambio necesario es aplicar la función exponencial $\exp (\bullet)^{1 / 1-\delta}$.

\section{Caso I $\quad \mathrm{d}=0$}

Aquí se tiene, de (3.1)

$$
\hat{Z}_{12 t+q}=\left(\bar{X} \bullet I_{q}\right)^{1 / 1-\delta}, \mathrm{t}=0,1, \ldots, \mathrm{m}-1 ; \mathrm{q}=1, \ldots, 12
$$

de donde

$$
\hat{Z}_{12 t+q}=Z_{12 m^{*} c-1}\left(\frac{I_{q}}{I c-1}\right)^{1 / 1 . \delta}, q=c, c+1, \ldots, 12
$$

Para suavizar el efecto que el fijar $\mathrm{Z}_{12 \mathrm{~m}+12}$ exógenamente ejerce sobre la distribución del financiamiento, se introduce un factor de discordancia $\varepsilon$, definido como el cociente entre $\mathrm{Z}_{12 \mathrm{~m}+12}$ determinado exógenamente en (3.2)- y su valor estimado por (3.4), o sea

$$
\varepsilon=\mathrm{Z}_{12 \mathrm{~m}+12} / \hat{Z}_{12 m+12}
$$

el cual se sugiere diluir en las estimaciones $\hat{Z}_{12 m+c}, \ldots \hat{Z}_{12 m+11}$, mediante la modificación de (3.4) a 


$$
\hat{Z}_{12 m+q}=\varepsilon^{\frac{q-c+1}{12-c+1}} Z_{12 m+c-1}\left(\frac{I_{q}}{I_{c-1}}\right)^{1 / 1-\delta}, q=c, c+1, \ldots, 11 .
$$

Con las relaciones (3.2), (3.5) y (3.6) es entonces posible realizar la distribución del financiamiento, como era requerido. Los siguientes dos casos $(\mathrm{d}=1 \mathrm{y} d=2)$, se resumen como modificaciones de las fórmulas (3.3), (3.4) y (3.6), las cuales se convierten en

Caso II $\quad \mathrm{d}=1$

$$
\begin{aligned}
& \hat{Z}_{12 t+q}=\left(\hat{Z}_{12 t+q-1}^{1-\delta}+\bar{X} \bullet I_{q}\right)^{1 / 1-\delta}, t=0,1, \ldots, m-1 ; q=1, \ldots, 12 \\
& \hat{Z}_{12 m+q}=\left(Z_{12 m+c-1}+\bar{X} \bullet \sum_{i=c}^{q} I_{i}\right)^{1 / 1-\delta}, q=c, c+1, \ldots, 12 \\
& \hat{Z}_{12 m+q}=\varepsilon^{\frac{q-c+1}{12-c+1}}\left(Z_{12 m+c-1}^{1-\delta}+\bar{X} \bullet \sum_{i=c}^{q} I_{i}\right)^{1 / 1-\delta}, q=c, c+1, \ldots, 11
\end{aligned}
$$

\section{Caso III $\mathrm{d}=2$}

$$
\begin{aligned}
& \hat{Z}_{12+q}=\left(2 \hat{Z}_{12 t+q-1}^{1-\delta}-\hat{Z}_{12 t+q-2}^{1-\delta}+\bar{X} I_{q}\right)^{1 / 1-\delta}, t=0,1, \ldots, m-1, \ldots, m-1 ; q=1, \ldots, 12 \\
& \hat{Z}_{12 m+q}=\left\{(q-c+2) Z_{12 m+c-1}^{1-\delta}-(q-c+1) Z_{12 m+c-2}^{1-\delta}+\bar{X}\left[I_{q}+2 I_{q-1}+\ldots+(q-c+1) I_{c}\right]\right\}^{1 / 1-\delta}, \\
& \mathrm{q}=\mathrm{c}, \mathrm{c}+1, \ldots, 12 \\
& \hat{Z}_{12 m+q}=\varepsilon^{\frac{q-c+1}{12-c+1}}\left\{(q-c+2) Z_{12 m+c-1}^{1-\delta}-(q-c+1) Z_{12 m+c-2}^{1-\delta}+\bar{X}\left[I_{q}+2 I_{q-1}+\ldots\right.\right. \\
& \left.\left.\quad \ldots+(q-c+1) I_{q}\right]\right\}^{1 / 1-q}, q=c, c+1, \ldots 11
\end{aligned}
$$

A continuación, y a manera de ejemplo, se aplicarán estas fórmulas al caso bajo estudio, para el cual se sabe que d=1. E $\bar{n}$ este caso particular se tiene, según (2.2) y (3.1), que debe usarse $\mathrm{X}^{\prime}=10^{-5} \bullet \bar{X}$ en lugar de $\bar{X}$, debido al factor utilizado para amplificar la escala.

Para juzgar la bondad de las estimaciones que se logran con este procedimiento, se realizará la distribución del financiamiento para los meses de mayo a diciembre de 1979. Así, se tiene $\mathrm{m}=9, \mathrm{c}=5$, 
$\mathrm{Z}_{112}=335583,5$ y $\mathrm{K}=87$ 025.8, de tal forma que $\mathrm{Z}_{120}=442$ 619.3. Además, se requiere conocer $\bar{X}{ }^{\prime}=10^{-}$ ${ }^{5}(-1.8299)$ y las sumas parciales $\sum_{i=c}^{q} I_{i}$ para q=c, c+1,..,12. Estos últimos valores se dan en la Tabla 6.

Tabla 6

Sumas Parciales de Índices Estacionales

\begin{tabular}{ccccccccc}
\hline & 5 & 6 & 7 & 8 & 9 & 10 & 11 & 12 \\
\hline$\sum_{i=5}^{q} I_{i}$ & 1.9441 & 3.2498 & 4.5024 & 5.5298 & 6.6708 & 7.5211 & 8.5338 & 9.4126 \\
\hline
\end{tabular}

Con esta información es posible calcular, primero $\hat{Z}_{120}=414110.53$ mediante (3.4'); acto seguido se obtiene $\varepsilon=Z_{120} / \hat{Z}_{120}=1.02054709$ y, entonces, la relación (3.6') proporciona las estimaciones que aparecen en la columna (1) de la Tabla 7. 
Tabla 7

Valores Estimados y observados del Financiamiento otorgado a la Banca Privada y Mixta durante 1979

\begin{tabular}{lccc}
\hline \multicolumn{4}{c}{ Financiamiento Otorgado a la Banca Privada y } \\
& \multicolumn{3}{c}{ Mixta } \\
\cline { 2 - 4 } & Estimado & Observado & Cociente \\
& $(1)$ & $(2)$ & $(3)=(1) /(2)$ \\
\hline Mayo & 350756.14 & 351782.2 & 0.9971 \\
Junio & 361803.18 & 361415.4 & 1.0011 \\
Julio & 372910.32 & 366874.3 & 1.0165 \\
Agosto & 382560.26 & 378330.3 & 1.0112 \\
Septiembre & 393583.08 & 387480.2 & 1.0158 \\
Octubre & 402352.51 & 401280.0 & 1.0027 \\
Noviembre & 412955.10 & 411639.3 & 1.0032 \\
\hline
\end{tabular}

De acuerdo con esta tabla, debe notarse que mientras más cercano a la unidad sea el cociente de la columna (3), mejor es el ajuste logrado. De aquí que se considere que el error sea menor del 1.7\%, puesto que ésta es la mayor divergencia observada (la cual ocurre en el mes de julio) y se afirme que el ajuste es adecuado..

\section{Conclusiones}

El presente análisis ha permitido corroborar, aunque sólo sea de manera empírica, la bondad del procedimiento introducido recientemente en Vera y Guerrero (1980). La experiencia obtenida con las pocas aplicaciones efectuadas a la fecha, conduce a pensar que el método puede ser equivalente a encontrar un filtro que convierta la serie bajo estudio en "ruido blanco" (en caso de que exista realmente un efecto estacional mensual). Falta, desde luego, fundamentar teóricamente esta hipótesis y obtener mayor evidencia empírica que la sustente.

Aunque el enfoque que se ha dado al método considera primordialmente la distribución del financiamiento, esto no debe ser restrictivo, puesto que su generalidad permite realizar el ajuste estacional de una serie, sin importar que ésta sea, o no, de financiamiento, siempre y cuando exista estacionalidad (quizá oculta por correlaciones espurias) en la serie. 
Por otro lado, respecto a la distribución del financiamiento, el procedimiento puede parecer demasiado complicado, si a final de cuentas lo que se desea es mantener el efecto estacional en las estimaciones futuras. Esto pudiera lograrse, quizá más fácilmente, pronosticando mediante la técnica de Box-Jenkins y ajustando los pronósticos por medio de un factor de discordancia entre lo obtenido de esta manera y lo deseado. La justificación del trabajo extra radica en la flexibilidad del método para manejar los índices estacionales, los cuales, por ejemplo, podrían tomarse como unitarios; de esta forma se eliminaría el efecto estacional en las estimaciones. 
REFERENCIAS

Bartlett, M.S. (1947), “The Use of Transformations”. Biometrika 3, 39-52.

Box, G.E.P. y Jenkins, G.M. (1970), Time Series Analysis, Forecasting and Control. Holden Dary.

Makridakis, S. y Whellwright, S.C. (1978), Forecasting Methods and Applications. John Wiley and Sons.

Vera, F.G. y Guerrero, G.V.M. (1980), Ajuste estacional de una serie de tiempo mediante el uso complemntario de métodos tradicionales y la técnica de Box-Jenkins. Banco de México, S.A., Subdirección de Investigación Económica, Documento de Investigación No. 22. 
BANCO DE MÉXICO

DIRECCIÓN GENERAL DE INVESTIGACIÓN ECONÓMICA

SERIE DOCUMENTOS DE INVESTIGACIÓN

1. ESTRUCTURA FINANCIERA Y EXPERIENCIA CAMBIARIA: MÉXICO 1954-1977.

Guillermo Ortíz

Octubre, 1978.

2. EL FINANCIAMIENTO DEL GASTO PUBLICO EN UNA ECONOMÍA EN CRECIMIENTO:

EL CASO DE MÉXICO.

Alain Ize

Noviembre, 1978.

3. ALGUNOS ASPECTOS DEL ENDEUDAMIENTO PÚBLICO EXTERNO EN MÉXCIO.

Ernesto Zedillo

Diciembre, 1978.

4. UNA APLICACIÓN DEL MODELO BAYESIANO DE DECISIÓN EN EL ANÁLISIS DE FUNCIONES DE PRODUCCIÓN AGRÍCOLA.

Héctor E. González Méndez.

Diciembre, 1978.

5. POLÍTICA MACROECONÓMICA EN EL CORTO PLAZO: UNA RESEÑA.

Alain Ize.

Marzo, 1979.

6. ESTUDIOS DE MONEDA Y BANCA Y POLÍTICA MONETARIA SOBRE MÉXICO:

SELECCIÓN BIBLIOGRÁFICA DE 1943 A 1978.

Abril, 1979.

7. COMERCIO EXTERIOR MÉXICO-ESTADOS UNIDOS: PROBLEMAS DE COMPARABILIDAD ESTADÍSTICA.

Jorge Carriles Rubio

Mayo, 1979.

8. EXPLOTACIÓN ÓPTIMA DE RESERVAS PETROLERAS EN UN CONTEXTO MACROECONÓMICO.

José Córdoba 
Mayo, 1979.

9. ASPECTOS DEFLACIONARIOS DE LA DEVALUACIÓN DEL PESO MEXICANO DE 1976. José Córdoba y Guillermo Ortíz

10. EXTRACCIÓN ÓPTIMA DE PETROLEO Y ENDEUDAMIENTO EXTERNO: EL CASO DE MÉXICO.

Ernesto Zedillo

Junio, 1979.

11. IMPUESTOS DIRECTOS: PROGRESIVIDAD ÓPTIMA.

Jesús Seade

Septiembre, 1979.

12. OPCIONES DE POLÍTICA ECONÓMICA 1979-1982.

Sócrates Rizzo y Leopoldo Solís

Septiembre, 1979.

13. INTERMEDIARIOS FINANCIEROS Y MERCADOS IMPERFECTOS DE CAPITAL.

Guillermo Ortíz

Septiembre, 1979

14. ESTIMACIONES DE EQUILIBRIO GENERAL DE LOS EFECTOS DE LAS DISTORSIONES EN LOS MERCADOS DE FACTORES: EL CASO DE MÉXICO.

José J. Sidaoui y Richard H. Sines

Octubre, 1979.

15. ANÁLISIS DE LA INFLACIÓN EN MÉXICO.

Alain Ize

Octubre, 1979.

16. ANÁLISIS DE LOS COMPONENTES DEL CAMBIO ESTRUCTURAL CON UN MODELO DE EQUILIBRIO GENERAL, 1970-75.

José J. Sidaoui y Richard H. Sines

Enero, 1980.

17. TIPOS DE CAMBIO FLOTANTES Y DESLIZ CAMBIARIO: LAS EXPERIENCIAS DE ALGUNOS PAÍSES EN DESARROLLO.

Guillermo Ortíz y Leopoldo Solís

Enero, 1980. 
18. UN MODELO DE INFLACIÓN Y CRECIMIENTO EN UNA ECONOMÍA CAPITALISTA EN DESARROLLO.

Alain Ize

Enero, 1980.

19. CRECIMIENTO E INFLACIÓN: ALTERNATIVAS CAMBIARIAS PARA MÉXICO.

Guillermo Ortíz y Leopoldo Solís

Febrero, 1980.

20. COMPORTAMIENTO DE LA CAPTACIÓN BANCARIA EN MÉXICO.

Héctor E. González Méndez

Mayo, 1980.

21. LA ENCUESTA DE TURISMO RECPETIVO. REPORTE METODOLÓGICO.

Alberto Vargas Aguayo

Junio, 1980.

22. AJUSTE ESTACIONAL DE UNA SERIE DE TIEMPO MEDIANTE EL USO

COMPLEMENTARIO DE MÉTODOS TRADICIONALES Y LA TÉCNICA DE BOXJENKINS.

Gabriel Vera Ferrer y Víctor M. Guerrero

Junio, 1980.

23. DISTRIBUCIÓN DEL FINANCIAMIENTO OTROGADO POR EL SISTEMA BANCARIO MEXICANO A LA BANCA PRIVADA Y MIXTA.

Víctor M. Guerrero y Gabriel Vera Ferrer

Julio, 1980. 